\title{
On the solvability of a boundary value problem on the real line
}

\author{
Giovanni Cupini ${ }^{1}$, Cristina Marcelli ${ }^{2}$ and Francesca Papalini ${ }^{2^{*}}$
}

\author{
* Correspondence: \\ papalini@dipmat.univpm.it \\ ${ }^{2}$ Dipartimento di Scienze \\ Matematiche - Università \\ Politecnica delle Marche, Via \\ Brecce Bianche, 60131 Ancona, \\ Italy \\ Full list of author information is \\ available at the end of the article
}

\begin{abstract}
We investigate the existence of heteroclinic solutions to a class of nonlinear differential equations

$$
\left(a(x) \Phi\left(x^{\prime}(t)\right)\right)^{\prime}=f\left(t, x(t), x^{\prime}(t)\right), \quad \text { a.e. } t \in \mathbb{R}
$$

governed by a nonlinear differential operator $\Phi$ extending the classical $p$-Laplacian, with right-hand side $f$ having the critical rate of decay -1 as $|t| \rightarrow+\infty$, that is $f(t, \cdot, \cdot) \approx \frac{1}{t}$. We prove general existence and non-existence results, as well as some simple criteria useful for right-hand side having the product structure $f(t, x, x)=b(t$, $x) c(x, x)$.

Mathematical subject classification: Primary: 34B40; 34C37; Secondary: 34B15; 34L30.

Keywords: boundary value problems, unbounded domains, heteroclinic solutions, nonlinear differential operators, $p$-Laplacian operator, $\Phi$ ?\$?-Laplacian operator
\end{abstract}

\section{Introduction}

Differential equations governed by nonlinear differential operators have been extensively studied in the last decade, due to their several applications in various sciences. The most famous differential operator is the well-known $p$-Laplacian and its generalization to the generic $\Phi$-Laplacian operator (an increasing homeomorphism of $\mathbb{R}$ with $\Phi(0)=0$ ). Many articles have been devoted to the study of differential equations of the type

$$
\left(\left(\Phi\left(x^{\prime}\right)\right)^{\prime}(t)=f\left(t, x(t), x^{\prime}(t)\right)\right.
$$

for $\Phi$-Laplacian operators, and recently also the study of singular or non-surjective differential operators has become object of an increasing interest (see, i.e., [1-10]).

On the other hand, in many applications the dynamic is described by a differential operator also depending on the state variable, like $\left(a(x) x^{\prime}\right)^{\prime}$ for some sufficiently regular function $a(x)$, which can be everywhere positive [non-negative] (as in the diffusion [degenerate] processes), or a changing sign function, as in the diffusion-aggregation models (see [7], [11-13]).

So, it naturally arises the interest for mixed nonlinear differential operators of the type $\left(a(x) \Phi\left(x^{\prime}\right)\right)^{\prime}$. In this context, in [11] we studied boundary value problems on the whole real line

(c) 2011 Cupini et al; licensee Springer. This is an Open Access article distributed under the terms of the Creative Commons Attribution License (http://creativecommons.org/licenses/by/2.0), which permits unrestricted use, distribution, and reproduction in any medium, provided the original work is properly cited. 


$$
\left\{\begin{array}{l}
\left(a(x(t)) \Phi\left(x^{\prime}(t)\right)\right)^{\prime}=f\left(t, x(t), x^{\prime}(t)\right) \\
x(-\infty)=v_{1}, \quad x(+\infty)=v_{2}
\end{array}\right.
$$

obtaining results on both existence and non-existence of heteroclinic solutions. Such criteria are based on the comparison between the behavior of the right-hand side $f(t, x$, $\left.x^{\prime}\right)$ as $|t| \rightarrow+\infty$ and $x^{\prime} \rightarrow 0$, combined to the infinitesimal order of the differential operator $\Phi\left(x^{\prime}\right)$ as $x^{\prime} \rightarrow 0$. Rather surprisingly, the presence of the state variable $x$ inside the right-hand side and the differential operator does not influence in any way the existence or the non-existence of solutions, but it only entails a more technical proof and a sligthly stronger set of assumptions on the operator $\Phi$. Roughly speaking, if $a(x)$ is positive and $f\left(t, x, x^{\prime}\right)=g\left(t, x^{\prime}\right) h(x)$ for some positive continuous function $h$, then the solvability of the boundary value problem depends neither on $a$, nor on $h$. Moreover, even the prescribed boundary values $v_{1}, v_{2}$ are not involved on the existence of solutions.

A crucial assumption in [11] is a limitation on the rate of the possible decay of $f(\cdot, x$, $x^{\prime}$ ) as $|t| \rightarrow+\infty$; precisely, we assumed that $f\left(t, x, x^{\prime}\right) \approx|t|^{\delta}$ for some $\delta>-1$ (possibly positive).

In the present article we focus our attention on right-hand sides having the critical rate of decay $\delta=-1$ and show that, contrary to the situation studied in [11], now the solvability of the boundary value problem is influenced by the behavior of the righthand side and of the differential operator with respect to the state variable $x$. For instance, when $f\left(t, x, x^{\prime}\right)=g(x) h\left(t, x^{\prime}\right)$ the existence of solutions depends on the amplitude of the range of the values assumed by the functions $a$ and $g$ in the interval $\left[v_{1}\right.$, $v_{2}$ ] determined by the prescribed boundary values.

In Section 2 we study the existence/non-existence of solutions for general right-hand sides $f\left(t, x(t), x^{\prime}(t)\right)$ (see Theorems 2.3-2.5); more operative criteria are stated in the subsequent section for $f$ of product type.

We conclude the article with some examples (see Examples 3.8-3.10), useful to have a quick glance on the role played by the behavior with respect to $x$.

The study of the solvability of the boundary value problem for rates of decay $\delta<-1$ is still open.

\section{Existence and non-existence theorems}

Let us consider the equation

$$
\left(a(x(t)) \Phi\left(x^{\prime}(t)\right)\right)^{\prime}=f\left(t, x(t), x^{\prime}(t)\right) \text { for a.e. } t \in \mathbb{R},
$$

where $a: \mathbb{R} \rightarrow \mathbb{R}$ is a positive continuous function, and $f: \mathbb{R}^{3} \rightarrow \mathbb{R}$ is a given Carathéodory function. From now on we will take into consideration increasing homeomorphisms $\Phi: \mathbb{R} \rightarrow \mathbb{R}$, with $\Phi(0)=0$.

Our approach is based on fixed point techniques suitably combined to the method of upper and lower solutions, according to the following definition.

Definition 2.1. A lower [upper] solution to equation (2.1) is a bounded function $\alpha \in$ $C^{1}(\mathbb{R})$ such that $(a \circ \alpha)\left(\Phi \circ \alpha^{\prime}\right) \in W^{1,1}(\mathbb{R})$ and

$$
\left(a(\alpha(t)) \Phi\left(\alpha^{\prime}(t)\right)\right)^{\prime} \geq[\leq] f\left(t, \alpha(t), \alpha^{\prime}(t)\right), \quad \text { for a.e. } t \in \mathbb{R} .
$$


Throughout this section we will assume the existence of an ordered pair of lower and upper solutions $\alpha$, $\beta$, i.e., satisfying $\alpha(t) \leq \beta(t)$ for every $t \in \mathbb{R}$, and we will adopt the following notations:

$$
\begin{aligned}
\mathcal{I} & \left.:=\inf _{t \in \mathbb{R}} \alpha(t), \sup _{t \in \mathbb{R}} \beta(t)\right], \quad v:=|\mathcal{I}|=\sup _{t \in \mathbb{R}} \beta(t)-\inf _{t \in \mathbb{R}} \alpha(t) \\
m & :=\min _{x \in \mathcal{I}} a(x)>0, \quad M:=\max _{x \in \mathcal{I}} a(x), \quad d:=\max \left\{\left|\alpha^{\prime}(t)\right|+\left|\beta^{\prime}(t)\right|: t \in \mathbb{R}\right\} .
\end{aligned}
$$

Note that the value $d$ is well-defined, in fact $\lim _{|t| \rightarrow+\infty} \alpha^{\prime}(t)=\lim _{|t| \rightarrow+\infty} \beta^{\prime}(t)=0$, since $(a \circ$ $\alpha)\left(\Phi \circ \alpha^{\prime}\right),(a \circ \beta)\left(\Phi \circ \beta^{\prime}\right)$ belong to $W^{1,1}(\mathbb{R})$ and $m>0$.

Moreover, in what follows $[x]^{+}$and $[x]^{-}$will respectively denote the positive and negative part of the real number $x$, and we set $x \wedge y:=\min \{x, y\}, x \vee y:=\max \{x, y\}$.

The next result proved in [11] concerns the convergence of sequences of functions correlated to solutions of the previous equation.

Lemma 2.2. For all $n \in \mathbb{N}$ let $I_{n}:=[-n, n]$ and let $u_{n} \in C^{1}\left(I_{n}\right)$ be such that: $\left(a \circ u_{n}\right)\left(\Phi \circ u_{n}^{\prime}\right) \in W^{1,1}\left(I_{n}\right)$, the sequences $\left(u_{n}(0)\right)_{n}$ and $\left(u_{n}^{\prime}(0)\right)_{n}$ are bounded and finally

$$
\left(a\left(u_{n}(t)\right) \Phi\left(u_{n}^{\prime}(t)\right)\right)^{\prime}=f\left(t, u_{n}(t), u_{n}^{\prime}(t)\right) \text { for a.e. } t \in I_{n} .
$$

Assume that there exist two functions $H, \gamma \in L^{1}(\mathbb{R})$ such that

$$
\left|u_{n}^{\prime}(t)\right| \leq H(t) \text { and }\left|a\left(u_{n}(t)\right) \Phi\left(u_{n}^{\prime}(t)\right)\right| \leq \gamma(t) \text { a.e. on } I_{n} \text {, for all } n \in \mathbb{N} \text {. }
$$

Then, the sequence $\left(x_{n}\right)_{n} \subset C^{1}(\mathbb{R})$ defined by

$$
x_{n}(t):=\left\{\begin{array}{l}
u_{n}(t) \quad \text { for } t \in I_{n} \\
u_{n}(n) \text { for } t>n \\
u_{n}(-n) \text { for } t<-n
\end{array}\right.
$$

admits a subsequence uniformly convergent in $\mathbb{R}$ to a function $x \in C^{1}(\mathbb{R})$, with $(a \circ x)$ $\left(\Phi \circ x^{\prime}\right) \in W^{1,1}(\mathbb{R})$, solution to equation $(2.1)$.

Moreover, if $\lim _{n \rightarrow+\infty} u_{n}(-n)=u^{-}$and $\lim _{n \rightarrow+\infty} u_{n}(n)=u^{+}$, then we have that

$$
\lim _{t \rightarrow-\infty} x(t)=u^{-} \quad \lim _{t \rightarrow+\infty} x(t)=u^{+}
$$

The first existence result concerns differential operators growing at most linearly at infinity.

Theorem 2.3. Assume that there exists a pair of lower and upper solutions $\alpha, \beta \in C^{1}$ $(\mathbb{R})$ of the equation (2.1), satisfying $\alpha(t) \leq \beta(t)$, for every $t \in \mathbb{R}$, with $\alpha$ increasing in $(-\infty$, $-L)$, $\beta$ increasing in $(L,+\infty)$, for some $L>0$.

Let $\Phi$ be such that

$$
\limsup _{|y| \rightarrow+\infty} \frac{|\Phi(\gamma)|}{|\gamma|}<+\infty
$$

and

$$
\liminf _{\gamma \rightarrow 0^{+}} \frac{\Phi(\gamma)}{\gamma^{\mu}}>0
$$

for some positive constant $\mu$.

Assume that there exist a constant $H>0$, a continuous function $\theta: \mathbb{R}^{+} \rightarrow \mathbb{R}^{+}$and a function $\lambda \in L^{q}([-L, L])$, with $1 \leq q \leq \infty$, such that 


$$
|f(t, x, y)| \leq \lambda(t) \theta(a(x)|\Phi(y)|) \quad \text { for a.e. }|t| \leq L, \text { every } x \in \mathcal{I},|y| \geq H
$$

$$
\int^{+\infty} \frac{\tau^{1-\frac{1}{q}}}{\theta(\tau)} \mathrm{d} \tau=+\infty
$$

(with $\frac{1}{q}=$ Oif $q=+\infty$ ).

Finally, suppose that for every $C>0$ there exist a function $\eta_{C} \in L^{1}(\mathbb{R})$ and a function $K_{C} \in W_{l o c}^{1,1}([0,+\infty))$, null in $[0, L]$ and strictly increasing in $[L,+\infty)$,

such that:

$$
\int^{+\infty} e^{-\frac{1}{\mu M} K_{C}(t)} \mathrm{d} t<+\infty
$$

and put

$$
N_{C}(t):=\Phi^{-1}\left(\frac{M}{m} \Phi(C) e^{-\frac{1}{M} K_{C}(|t|)}\right)
$$

we have

$$
\begin{aligned}
& \left\{\begin{array}{l}
f(t, x, y) \leq-K^{\prime} C(t) \Phi(|y|) \\
f(-t, x, y) \geq K^{\prime} C(t) \Phi(|y|)
\end{array} \text { for a.e. } t \geq L, \quad \text { every } x \in \mathcal{I}, \quad|y| \leq N_{C}(t),\right. \\
& |f(t, x, y)| \leq \eta_{C}(t) \quad \text { if } x \in \mathcal{I},|y| \leq N_{C}(t)+\left|\alpha^{\prime}(t)\right|+\left|\beta^{\prime}(t)\right|, \text { for a.e. } t \in \mathbb{R} .
\end{aligned}
$$

Then, there exists a function $x \in C^{1}(\mathbb{R})$, with $(a \circ x)\left(\Phi \circ x^{\prime}\right) \in W^{1,1}(\mathbb{R})$, such that

$$
\begin{cases}\left(a(x(t)) \Phi\left(x^{\prime}(t)\right)\right)^{\prime}=f\left(t, x(t), x^{\prime}(t)\right) & \text { for a.e. } t \in \mathbb{R} \\ \alpha(t) \leq x(t) \leq \beta(t) & \text { for every } t \in \mathbb{R} \\ x(-\infty)=\alpha(-\infty), x(+\infty)=\beta(+\infty) & \end{cases}
$$

Proof. In some parts the proof is similar to that of Theorem 3.2 [11]. So, we provide here only the arguments which differ from those used in that proof.

By (2.2), without loss of generality we assume $H>\frac{v}{2 L}$ and

$$
|\Phi(\gamma)| \leq K|y| \quad \text { whenever }|y|>H,
$$

for some constant $K>0$.

Moreover, by (2.5), there exists a constant $C>\Phi^{-1}\left(\frac{M}{m} \Phi(H)\right) \geq H$ such that

$$
\int_{M \Phi(H)}^{m \Phi(C)} \frac{\tau^{1-\frac{1}{q}}}{\theta(\tau)} \mathrm{d} \tau>(K M \nu)^{1-\frac{1}{q}}\|\lambda\|_{q}
$$

Fix $n \in \mathbb{N}, n>L$, and put $I_{n}:=[-n, n]$.

Let us consider the following auxiliary boundary value problem on the compact interval $I_{n}$ :

$$
\left(P_{n}^{*}\right)\left\{\begin{array}{l}
\left(a\left(T_{x}(t)\right) \Phi\left(x^{\prime}(t)\right)\right)^{\prime}=f\left(t, T_{x}(t), Q_{x}(t)\right)+\arctan (w(t, x(t))), \quad \text { a.e. } t \in I_{n} \\
x(-n)=\alpha(-n), \quad x(n)=\beta(n)
\end{array}\right.
$$


where $T: W^{1,1}\left(I_{n}\right) \rightarrow W^{1,1}\left(I_{n}\right)$ is the truncation operator defined by

$$
\begin{gathered}
T_{x}(t):=[\beta(t) \wedge x(t)] \vee \alpha(t) ; \\
Q_{x}(t):=-\left(N_{C}(t)+\left|\alpha^{\prime}(t)\right|+\left|\beta^{\prime}(t)\right|\right) \vee\left[T^{\prime}(t) \wedge\left(N_{C}(t)+\left|\alpha^{\prime}(t)\right|+\left|\beta^{\prime}(t)\right|\right)\right] ;
\end{gathered}
$$

and finally $w: \mathbb{R}^{2} \rightarrow \mathbb{R}$ is the penalty function defined by $w(t, x):=[x-\beta(t)]^{+}-[x-\alpha$ $(t)]^{-}$.

By the same argument used in the proof of Theorem 3.2 [11], one can show, using only assumption (2.9), that for every $n>L$ problem $\left(P_{n}^{*}\right)$ admits a solution $u_{n}$ such that

$$
\alpha(t) \leq u_{n}(t) \leq \beta(t) \quad \text { for all } t \in I_{n},
$$

hence $T_{u_{n}}(t) \equiv u_{n}(t)$ and $w\left(t, u_{n}(t)\right) \equiv 0$. Moreover, it is possible to prove that

$$
\begin{aligned}
& u_{n}^{\prime}(t) \geq 0 \quad \text { whenever } L \leq|t| \leq n \\
& u_{n}^{\prime}\left(t_{0}\right)=0 \text { for some } t_{0} \in[L, n) \quad \Rightarrow \quad u_{n}^{\prime}(t) \equiv 0 \text { in }\left[t_{0}, n\right)
\end{aligned}
$$

(see Steps 3 and 4 in the proof of Theorem 3.2 [11]).

Now our goal is to prove an a priori bound for the derivatives, that is $\left|u_{n}^{\prime}(t)\right| \leq N_{C}(t)$ for a.e. $t \in I_{n}$. We split this part into two steps.

Step 1. We have $\left|u_{n}^{\prime}(t)\right|<C \leq N_{C}(t)$ for every $t \in[-L, L]$.

Indeed, since $u_{n} \in C^{1}\left(I_{n}\right)$ and $u_{n}([-L, L]) \subset \mathcal{I}$, we can apply Lagrange Theorem to deduce that for some $\tau_{0} \in[-L, L]$ we have

$$
\left|u_{n}^{\prime}\left(\tau_{0}\right)\right|=\frac{1}{2 L}\left|u_{n}(L)-u_{n}(-L)\right| \leq \frac{\sup \beta-\inf \alpha}{2 L}=\frac{v}{2 L}<H<C .
$$

Assume, by contradiction, the existence of an interval $\left(\tau_{1}, \tau_{2}\right) \subset(-L, L)$ such that $H<\left|u_{n}^{\prime}(t)\right|<C$ in $\left(\tau_{1}, \tau_{2}\right)$ and $\left|u_{n}^{\prime}\left(\tau_{1}\right)\right|=H,\left|u_{n}^{\prime}\left(\tau_{2}\right)\right|=C$ or viceversa.

Since $N_{C}(t)=\Phi^{-1}\left(\frac{M}{m} \Phi(C)\right) \geq C$ for every $t \in\left(\tau_{1}, \tau_{2}\right)$, we have $\left|u_{n}^{\prime}(t)\right|<N_{C}(t)$ for every $t \in\left(\tau_{1}, \tau_{2}\right)$. Then, by the definition of $\left(P_{n}^{*}\right)$ and assumption (2.4), for a.e. $t \in\left(\tau_{1}\right.$, $\tau_{2}$ ) we have

$$
\begin{aligned}
& \left|\left(a\left(u_{n}(t)\right) \Phi\left(u_{n}^{\prime}(t)\right)\right)^{\prime}\right|=\left|\left(a\left(T_{u_{n}}(t)\right) \Phi\left(u_{n}^{\prime}(t)\right)\right)^{\prime}\right|=\left|f\left(t, T_{u_{n}}(t), Q_{u_{n}}(t)\right)\right| \\
& =\left|f\left(t, u_{n}(t), u_{n}^{\prime}(t)\right)\right| \leq \lambda(t) \theta\left(a\left(u_{n}(t)\right)\left|\Phi\left(u_{n}^{\prime}(t)\right)\right|\right) .
\end{aligned}
$$

Therefore, using a change of variable and the Hölder inequality, we get

$$
\begin{aligned}
& \int_{M \Phi(H)}^{m \Phi(C)} \frac{\tau^{1-\frac{1}{q}}}{\theta(\tau)} \mathrm{d} \tau \leq \int_{\tau_{1}}^{\tau_{2}} \frac{\left|a\left(u_{n}(t)\right) \Phi\left(u_{n}^{\prime}(t)\right)\right|^{1-\frac{1}{q}}}{\theta\left(\left|a\left(u_{n}(t)\right) \Phi\left(u_{n}^{\prime}(t)\right)\right|\right)}\left|\left(a\left(u_{n}(t)\right) \Phi\left(u_{n}^{\prime}(t)\right)\right)^{\prime}\right| \mathrm{d} t \\
& \leq \int_{\tau_{1}}^{\tau_{2}} \lambda(t)\left|a\left(u_{n}(t)\right) \Phi\left(u_{n}^{\prime}(t)\right)\right|^{1-\frac{1}{q}} \mathrm{~d} t \leq\|\lambda\|_{q}\left(M \int_{\tau_{1}}^{\tau_{2}}\left|\Phi\left(u_{n}^{\prime}(t)\right)\right| \mathrm{d} t\right)^{1-\frac{1}{q}} .
\end{aligned}
$$

Moreover, since $u_{n}^{\prime}$ has constant sign in $\left(\tau_{1}, \tau_{2}\right)$, using (2.12) we have

$$
\int_{\tau_{1}}^{\tau_{2}}\left|u_{n}^{\prime}(t)\right| \mathrm{d} t=\left|u_{n}\left(\tau_{2}\right)-u_{n}\left(\tau_{1}\right)\right| \leq v .
$$

Therefore, by (2.10), from the previous chain of inequalities we deduce

$$
\int_{M \Phi(H)}^{m \Phi(C)} \frac{\tau^{1-\frac{1}{q}}}{\theta(\tau)} \mathrm{d} \tau \leq\|\lambda\|_{q}\left(K M \int_{\tau_{1}}^{\tau_{2}}\left|u_{n}^{\prime}(t)\right| \mathrm{d} t\right)^{1-\frac{1}{q}} \leq\|\lambda\|_{q}(K M \nu)^{1-\frac{1}{q}}
$$


in contradiction with (2.11). Thus, we get $\left|u_{n}^{\prime}(t)\right|<C$ for every $t \in[-L, L]$ and the claim is proved.

Step 2. We have $u_{n}^{\prime}(t)<N_{C}(t)$ for every $t \in I_{n} \backslash[-L, L]$.

Define $\hat{t}:=\sup \left\{t>L: u_{n}^{\prime}(\tau)<N_{C}(\tau)\right.$ for every $\left.\tau \in[L, t]\right\}$, and assume by contradiction that $\hat{t}<n$. Hence, $u_{n}^{\prime}(\hat{t})=N_{C}(\hat{t})>0$ and by (2.13), (2.14) we deduce that $u_{n}^{\prime}(t)>0$ in $[L, \hat{t}]$. Moreover, by (2.12) and the definition of $Q_{u_{n}}$ we get

$$
\left(a\left(u_{n}(t)\right) \Phi\left(u_{n}^{\prime}(t)\right)\right)^{\prime}=f\left(t, u_{n}(t), u_{n}^{\prime}(t)\right) \text { in }[L, \hat{t}],
$$

so, by (2.8) we have

$$
\left(a\left(u_{n}(t)\right) \Phi\left(u_{n}^{\prime}(t)\right)\right)^{\prime} \leq-K_{C}^{\prime}(t) \Phi\left(u_{n}^{\prime}(t)\right) \leq-\frac{K_{C}^{\prime}(t)}{M} a\left(u_{n}(t)\right) \Phi\left(u_{n}^{\prime}(t)\right), \quad \text { a.e. in }[L, \hat{t}] .
$$

Then, recalling that $K_{C}(L)=0$ and $u_{n}^{\prime}(t)>0$ for every $t \in[L, \hat{t}]$, we infer

$$
\frac{a\left(u_{n}(t)\right) \Phi\left(u_{n}^{\prime}(t)\right)}{a\left(u_{n}(L)\right) \Phi\left(u_{n}^{\prime}(L)\right)}=e^{\int_{L}^{t} \frac{\left(a\left(u_{n}(s)\right) \Phi\left(u_{n}^{\prime}(s)\right)\right)^{\prime}}{a\left(u_{n}(s)\right) \Phi\left(u_{n}^{\prime}(s)\right)} \mathrm{d} s} \leq e^{-\frac{1}{M} K_{C}(t)}
$$

implying

$$
a\left(u_{n}(t)\right) \Phi\left(u_{n}^{\prime}(t)\right) \leq a\left(u_{n}(L)\right) \Phi\left(u_{n}^{\prime}(L)\right) e^{-\frac{1}{M} K_{C}(t)}<M \Phi(C) e^{-\frac{1}{M} K_{C}(t)}
$$

since $u_{n}^{\prime}(L)<C$. Therefore, $u_{n}^{\prime}(t) \leq N_{C}(t)$ for every $t \in[L, \hat{t}]$, in contradiction with the definition of $\hat{t}$. The same argument works in the interval $[-n,-L]$ and the claim is proved.

Summarizing, since $\left|u_{n}^{\prime}(t)\right| \leq N_{C}(t)$ for every $t \in I_{n}$, by the definition of $Q_{u_{n}}$ we have

$$
\left(a\left(u_{n}(t)\right) \Phi\left(u_{n}^{\prime}(t)\right)\right)^{\prime}=f\left(t, u_{n}(t), u_{n}^{\prime}(t)\right) \quad \text { for a.e. } t \in I_{n} .
$$

Observe now that condition (2.3) implies that $\limsup _{\xi \rightarrow 0^{+}} \frac{\Phi^{-1}(\xi)}{\xi^{1 / \mu}}<+\infty$. Hence, by assumption (2.6) we get $N_{C} \in L^{1}(\mathbb{R})$ and applying Lemma 2.2 with $H(t)=N_{C}(t)$ and $\gamma$ $(t)=\eta_{C}(t)$ we deduce the existence of a solution $x$ to problem $(P)$.

In order to deal with differential operators having superlinear growth at infinity, we need to strengthen condition (2.5), taking a Nagumo function with sublinear growth at infinity, as in the statement of the following result.

Theorem 2.4. Suppose that all the assumptions of Theorem 2.3 are satisfied, with the exception of (2.2), and with (2.5) replaced by

$$
\lim _{y \rightarrow+\infty} \frac{\theta(\gamma)}{y}=0 .
$$

Then, the assertion of Theorem 2.3 follows.

Proof. The proof is quite similar to that of the previous Theorem. Indeed, notice that assumptions (2.2) and (2.5) of Theorem 2.3 have been used only in the choice of the constant $C$ (see (2.11)) and in the proof of Step 1. Hence, we now present only the proof of this part, the rest being the same. 
Notice that by assumption (2.17), we have

$$
\lim _{\xi \rightarrow+\infty} \frac{\int_{M \Phi(H)}^{m \xi} \frac{\tau^{1-\frac{1}{q}}}{\theta(\tau)} \mathrm{d} \tau}{\xi^{1-\frac{1}{q}}}=+\infty
$$

hence, there exists a constant $C>\Phi^{-1}\left(\frac{M}{m} \Phi(H)\right) \geq H$ such that

$$
\int_{M \Phi(H)}^{m \Phi(C)} \frac{\tau^{1-\frac{1}{q}}}{\theta(\tau)} \mathrm{d} \tau>(2 M L \Phi(C))^{1-\frac{1}{q}}\|\lambda\|_{q} .
$$

With this choice of the constant $C$, the proof proceeds as in Theorem 2.3. The only modification concerns formula (2.16), which becomes, taking (2.15) into account:

$$
\int_{M \Phi(H)}^{m \Phi(C)} \frac{\tau^{1-\frac{1}{q}}}{\theta(\tau)} \mathrm{d} \tau \leq\|\lambda\|_{q}\left(M \int_{\tau_{1}}^{\tau_{2}}\left|\Phi\left(u_{n}^{\prime}(t)\right)\right| \mathrm{d} t\right)^{1-\frac{1}{q}} \leq\|\lambda\|_{q}(2 M L \Phi(C))^{1-\frac{1}{q}}
$$

in contradiction with (2.18). From here on, the proof proceeds in the same way. $\square$

In the particular case of $p$-Laplacian operators, one can use the positive homogeneity for weakening assumption (2.17) of Theorem 2.4 and widening the class of the admissible Nagumo functions, as we show in the following result.

Theorem 2.5. Let $\Phi: \mathbb{R} \rightarrow \mathbb{R}, \Phi(y)=|y|^{p-2} y$, and assume that there exists a pair of lower and upper solutions $\alpha, \beta \in C^{1}(\mathbb{R})$ to equation (2.1), satisfying $\alpha(t) \leq \beta(t)$, for every $t \in \mathbb{R}$, with $\alpha$ increasing in $(-\infty,-L), \beta$ increasing in $(L,+\infty)$, for some constant $L$ $>0$.

Moreover, assume that there exist a positive constant $H$, a continuous function $\theta: \mathbb{R}^{+} \rightarrow \mathbb{R}^{+}$and a function $\lambda \in L^{q}([-L, L])$, with $1 \leq q \leq+\infty$, such that

$$
\begin{aligned}
& |f(t, x, y)| \leq \lambda(t) \theta\left(a(x)|y|^{p-1}\right) \quad \text { for a.e. }|t| \leq L, \text { every } x \in \mathcal{I}, \quad|y| \geq H \\
& \int^{+\infty} \frac{\tau^{\frac{1}{p-1}\left(1-\frac{1}{q}\right)}}{\theta(\tau)} \mathrm{d} \tau=+\infty .
\end{aligned}
$$

Finally, suppose that for every $C>0$ there exist a function $\eta_{C} \in L^{1}(\mathbb{R})$ and a function $K_{C} \in W_{\text {loc }}^{1,1}([0,+\infty))$, null in $[0, L]$ and strictly increasing in $[L,+\infty)$, such that:

$$
\int^{+\infty} e^{-\frac{1}{M(p-1)} K_{C}(t)} \mathrm{d} t<+\infty
$$

and put

$$
N_{C}(t):=C\left(\frac{M}{m}\right)^{\frac{1}{p-1}} e^{-\frac{1}{M(p-1)} K_{C}(|t|)}
$$

we have

$$
\left\{\begin{array}{l}
f(t, x, y) \leq-K^{\prime} C(t)|y|^{p-1} \\
f(-t, x, y) \geq K_{C}^{\prime}(t)|y|^{p-1}
\end{array} \text { for a.e. } t \geq L, \text { every } x \in \mathcal{I}, \quad|y| \leq N_{C}(t),\right.
$$




$$
|f(t, x, y)| \leq \eta_{C}(t) \quad \text { if } x \in \mathcal{I},|y| \leq N_{C}(t)+\left|\alpha^{\prime}(t)\right|+\left|\beta^{\prime}(t)\right|, \quad \text { for a.e. } t \in \mathbb{R} \text {. (2.23) }
$$

Then, there exists a function $x \in C^{1}(\mathbb{R})$, with $(a \circ x)\left(\Phi \circ x^{\prime}\right) \in W^{1,1}(\mathbb{R})$, such that

$$
\begin{cases}\left(a(x(t)) \Phi\left(x^{\prime}(t)\right)\right)^{\prime}=f\left(t, x(t), x^{\prime}(t)\right) & \text { for a.e. } t \in \mathbb{R} \\ \alpha(t) \leq x(t) \leq \beta(t) & \text { for every } t \in \mathbb{R} \\ x(-\infty)=\alpha(-\infty), x(+\infty)=\beta(+\infty) & \end{cases}
$$

Proof. The proof is quite similar to that of Theorem 2.3. Indeed, notice that the present statement has the same assumptions of Theorem 2.3, written for $\Phi(y)=|y|^{p-2} y$, with the exception of conditions (2.2) and (2.5), which were used only in the proof of Step 1. Hence, as in the proof of the previous Theorem 2.4, we now provide only the proof of Step 1, the rest being the same.

At the beginning of the proof, without loss of generality we assume $H>\frac{v}{2 L}$ and we choose $C>\left(\frac{M}{m}\right)^{\frac{1}{p-1}} H \geq H$, in such a way that

$$
\int_{M H^{p-1}}^{m C^{p-1}} \frac{\tau^{\frac{1}{p-1}\left(1-\frac{1}{q}\right)}}{\theta(\tau)} \mathrm{d} \tau>\|\lambda\|_{q}\left(v M^{\frac{1}{p-1}}\right)^{1-\frac{1}{q}} .
$$

The proof of Step 1 begins as previously, determining an interval $J=\left(\tau_{1}, \tau_{2}\right) \subset(-L, L)$ such that $\left|u_{n}^{\prime}\left(\tau_{0}\right)\right|=\frac{1}{2 L}\left|u_{n}(L)-u_{n}(-L)\right| \leq \frac{\sup \beta-\inf \alpha}{2 L}=\frac{v}{2 L}<H<C$. in $J$, and $\left|u_{n}^{\prime}\left(\tau_{2}\right)\right|=C,\left|u_{n}^{\prime}\left(\tau_{2}\right)\right|=C$ or vice versa. Then, as in the proof of Theorem 2.3, assumption (2.19) implies that for a.e. $t \in J$ we have

$$
\begin{aligned}
& \left|\left(a\left(u_{n}(t)\right) \Phi\left(u_{n}^{\prime}(t)\right)\right)^{\prime}\right|=\left|\left(a\left(T_{u_{n}}(t)\right) \Phi\left(u_{n}^{\prime}(t)\right)\right)^{\prime}\right|=\left|f\left(t, T_{u_{n}}(t), Q_{u_{n}}(t)\right)\right|= \\
& =\left|f\left(t, u_{n}(t), u_{n}^{\prime}(t)\right)\right| \leq \lambda(t) \theta\left(a\left(u_{n}(t)\right)\left|u_{n}^{\prime}(t)\right|^{p-1}\right) .
\end{aligned}
$$

Therefore, put

$$
\alpha_{1}:=a\left(x\left(\tau_{1}\right)\right)\left|x^{\prime}\left(\tau_{1}\right)\right|^{p-1}, \quad \alpha_{2}:=a\left(x\left(\tau_{2}\right)\right)\left|x^{\prime}\left(\tau_{2}\right)\right|^{p-1},
$$

we get

$$
\begin{aligned}
& \int_{M H^{p-1}}^{m C^{p-1}} \frac{\tau^{\frac{1}{p-1}\left(1-\frac{1}{q}\right)}}{\theta(\tau)} \mathrm{d} \tau \leq\left|\int_{\alpha_{1}}^{\alpha_{2}} \frac{\tau^{\frac{1}{p-1}\left(1-\frac{1}{q}\right)}}{\theta(\tau)} \mathrm{d} \tau\right| \\
& =\left|\int_{\tau_{1}}^{\tau_{2}} \frac{\left(a\left(u_{n}(t)\right)\left|u_{n}^{\prime}(t)\right|^{p-1}\right)^{\frac{1}{p-1}\left(1-\frac{1}{q}\right)}}{\theta\left(a\left(u_{n}(t)\right)\left|u_{n}^{\prime}(t)\right|^{p-1}\right)}\right|\left(a\left(u_{n}(t)\right)\left|u_{n}^{\prime}(t)\right|^{p-1}\right)^{\prime}|\mathrm{d} t| \\
& \leq \int_{\tau_{1}}^{\tau_{2}} \lambda(t)\left(a\left(u_{n}(t)\right)^{\frac{1}{p-1}}\left|u_{n}^{\prime}(t)\right|\right)^{1-\frac{1}{q}} \mathrm{~d} t \\
& \leq\|\lambda\|_{q} M^{\frac{1}{p-1}\left(1-\frac{1}{q}\right)}\left(\int_{\tau_{1}}^{\tau_{2}}\left|u_{n}^{\prime}(t)\right| \mathrm{d} t\right)^{1-\frac{1}{q}} \leq\|\lambda\|_{q}\left(v M^{\frac{1}{p-1}}\right)^{1-\frac{1}{q}}
\end{aligned}
$$

in contradiction with (2.24). Thus, we get $\left|u_{n}^{\prime}(t)\right|<C$ for every $t \in[-L, L]$ and Step 1 is proved.

As we mentioned in Section 1, the assumptions of the previous existence Theorems are not improvable in the sense that if conditions (2.3) and (2.8) are satisfied with the reversed inequalities and the summability condition (2.6) [respectively (2.21) for the 
case of $p$-Laplacian] does not hold, then problem $(P)$ does not admit solutions, as the following results state.

Theorem 2.6. Suppose that

$$
\limsup _{y \rightarrow 0^{+}} \frac{\Phi(\gamma)}{\gamma^{\mu}}<+\infty
$$

for some positive constant $\mu$. Moreover, assume that there exist two constants $L \geq 0, \rho$ $>0$ and a positive strictly increasing function $K \in W_{\text {loc }}^{1,1}([L,+\infty))$ satisfying

$$
\int^{+\infty} e^{-\frac{1}{\mu \tilde{m}} K(t)} \mathrm{d} t=+\infty
$$

where $\tilde{m}:=\min _{x \in\left[v^{-}, \nu^{+}\right]} a(x)$, such that one of the following pair of conditions holds:

$$
f(t, x, y) \geq-K^{\prime}(t) \Phi(|y|) \quad \text { for a.e. } t \geq L, \text { every } x \in\left[\nu^{-}, \nu^{+}\right],|y|<\rho
$$

or

$$
f(t, x, y) \leq K^{\prime}(-t) \Phi(|y|) \quad \text { for a.e. } t \leq-L, \quad \text { every } x \in\left[v^{-}, v^{+}\right],|y|<\rho .
$$

Moreover, assume that

$$
\operatorname{tf}(t, x, y) \leq 0 \quad \text { for a.e. }|t| \geq L, \quad \text { every } x \in \mathbb{R},|y|<\rho .
$$

Then, problem $(P)$ can only admit solutions which are constant in $[L,+\infty)$ (when (2.27) holds) or constant in $(-\infty,-L]$ (when (2.28) holds). Therefore, if both (2.27) and (2.28) hold and $L=0$, then problem $(P)$ does not admit solutions. More precisely, no function $x \in C^{1}(\mathbb{R})$, with $(a \circ x)\left(\Phi \circ x^{\prime}\right)$ almost everywhere differentiable, exists satisfying the boundary conditions and the differential equation in $(P)$.

Proof. Suppose that (2.27) holds (the proof is the same if (2.28) holds).

Let $x \in C^{1}(\mathbb{R})$, with $(a \circ x)\left(\Phi \circ x^{\prime}\right)$ almost everywhere differentiable (not necessarily belonging to $W^{1,1}(\mathbb{R})$ ), be a solution of problem $(P)$. First of all, let us prove that $\lim _{t \rightarrow+\infty} \Phi\left(x^{\prime}(t)\right)=0$.

Indeed, since $x(+\infty)=v^{+} \in \mathbb{R}$, we have $\limsup _{t \rightarrow+\infty} x^{\prime}(t) \geq 0$ and $\liminf _{t \rightarrow+\infty} x^{\prime}(t) \leq 0$.

Taking into account that $\Phi$ is an increasing homeomorphism with $\Phi(0)=0$, if $\liminf _{t \rightarrow+\infty} x^{\prime}(t)<0$, then there exists an interval $\left[t_{1}, t_{2}\right] \subset[L,+\infty)$ such that $-\rho<\Phi\left(x^{\prime}(t)\right)$ $<0$ in $\left[t_{1}, t_{2}\right], \Phi\left(x^{\prime}\left(t_{2}\right)\right)>\frac{m}{M} \Phi\left(x^{\prime}\left(t_{1}\right)\right)$. But by virtue of assumption (2.29)

we deduce that $a(x(t)) \Phi\left(x^{\prime}(t)\right)$ is decreasing in $\left[t_{1}, t_{2}\right]$ and then

$$
\Phi\left(x^{\prime}\left(t_{2}\right)\right) \leq \frac{1}{M} a\left(x\left(t_{2}\right)\right) \Phi\left(x^{\prime}\left(t_{2}\right)\right) \leq \frac{1}{M} a\left(x\left(t_{1}\right)\right) \Phi\left(x^{\prime}\left(t_{1}\right)\right) \leq \frac{m}{M} \Phi\left(x^{\prime}\left(t_{1}\right)\right),
$$

a contradiction. Hence, necessarily $\lim _{t \rightarrow+\infty} \inf ^{\prime}(t)=0$. We can prove in a similar way that $\limsup _{t \rightarrow+\infty} x^{\prime}(t)=0$. So, $\lim _{t \rightarrow+\infty} x^{\prime}(t)=0$ and we can define $t^{*}:=\inf \left\{t \geq L:\left|x^{\prime}(\tau)\right|<\rho\right.$ in $[t,+\infty)\}$.

We claim that $x^{\prime}(t) \geq 0$ for every $t \geq t^{*}$. Indeed, if $x^{\prime}(\hat{t})<0$ for some $\hat{t} \geq t^{*}$, since $a(x$ $(t)) \Phi\left(x^{\prime}(t)\right)$ is decreasing in $\left[t^{*},+\infty\right)$ by $(2.29)$, we get

$$
a(x(t)) \Phi\left(x^{\prime}(t)\right) \leq a(x(\hat{t})) \Phi\left(x^{\prime}(\hat{t})\right) \leq m \Phi\left(x^{\prime}(\hat{t})\right)<0, \quad \text { for every } t \geq \hat{t} .
$$


Since $a$ is positive, then $\Phi\left(x^{\prime}(t)\right)<0$ for every $t \geq \hat{t}$. Hence, from (2.30) we get $M \Phi\left(x^{\prime}(t)\right) \leq m \Phi\left(x^{\prime}(\hat{t})\right)$, and so

$$
x^{\prime}(t) \leq \Phi^{-1}\left(\frac{m}{M} \Phi\left(x^{\prime}(\hat{t})\right)\right)<0 \quad \text { for every } t \geq \hat{t}
$$

in contradiction with the boundedness of $x$. Thus, the claim is proved.

Let us define $\tilde{t}:=\inf \left\{t \geq t^{*}: x(\tau) \geq v^{-}\right.$in $\left.[t,+\infty)\right\} \geq t^{*}$. We now prove that $x^{\prime}(t)=0$ for every $t \geq \tilde{t}$.

Let us assume by contradiction that $x^{\prime}(\bar{t})>0$ for some $\bar{t} \geq \tilde{t}$. Put $T:=\sup \left\{t \geq \bar{t}: x^{\prime}(\tau)>0\right.$ in $\left.[\bar{t}, t]\right\}$; we claim that $T=+\infty$. Indeed, if $T<+\infty$, since $0<$ $x^{\prime}(t)<\rho$ in $[\bar{t}, T]$, by $(2.27)$ we have

$$
\left(a(x(t)) \Phi\left(x^{\prime}(t)\right)\right)^{\prime}=f\left(t, x(t), x^{\prime}(t)\right) \geq-K^{\prime}(t) \Phi\left(x^{\prime}(t)\right) \text { for a.e. } t \in[\bar{t}, T] .
$$

So, assuming without loss of generality $\rho \leq 1$, we get

$$
\left(a(x(t)) \Phi\left(x^{\prime}(t)\right)\right)^{\prime} \geq-K^{\prime}(t) \Phi\left(x^{\prime}(t)\right) \geq-\frac{K^{\prime}(t)}{\bar{m}} a(x(t)) \Phi\left(x^{\prime}(t)\right)
$$

where $\bar{m}:=\min _{\xi \in[x(\bar{t}), x(T)]} a(\xi)$. Then, integrating in $[t, T]$ with $t<T$ we obtain (taking into account that $x^{\prime}(T)=0$ )

$$
a(x(t)) \Phi\left(x^{\prime}(t)\right) \leq \int_{t}^{T} \frac{K^{\prime}(\tau)}{\bar{m}} a(x(\tau)) \Phi\left(x^{\prime}(\tau)\right) \mathrm{d} \tau \quad \text { for every } t \in(\bar{t}, T]
$$

so by the Gronwall's inequality we deduce $a(x(t)) \Phi\left(x^{\prime}(t)\right) \leq 0$, i.e. $x^{\prime}(t) \leq 0$ in the same interval, in contradiction with the definition of $T$. Hence $T=+\infty$.

Therefore, since $0<x^{\prime}(t)<\rho$ and $v^{-} \leq x(t) \leq v^{+}$in $[\bar{t},+\infty)$, we get

$$
\left(a(x(t)) \Phi\left(x^{\prime}(t)\right)\right)^{\prime}=f\left(t, x(t), x^{\prime}(t)\right) \geq-K^{\prime}(t) \Phi\left(x^{\prime}(t)\right) \geq \frac{-K^{\prime} C(t)}{\tilde{m}} a(x(t)) \Phi\left(x^{\prime}(t)\right)
$$

for a.e.t $\geq \bar{t}$, where $\tilde{m}:=\min _{x \in\left[v^{-}, v^{+}\right]} a(x)$. The above inequalities imply that for a.e. $t \geq \bar{t}$

$$
\log \frac{a(x(t)) \Phi\left(x^{\prime}(t)\right)}{a(x(\bar{t})) \Phi\left(x^{\prime}(\bar{t})\right)}=\int_{\bar{t}}^{t} \frac{\left(a(x(s)) \Phi\left(x^{\prime}(s)\right)\right)^{\prime}}{a(x(s)) \Phi\left(x^{\prime}(s)\right)} \mathrm{d} s \geq \frac{1}{\tilde{m}}(K(\bar{t})-K(t))
$$

and then

$$
\Phi\left(x^{\prime}(t)\right) \geq \frac{1}{\tilde{M}} a(x(\bar{t})) \Phi\left(x^{\prime}(\bar{t})\right) e^{\frac{1}{\tilde{m}}(K(\bar{t})-K(t))}
$$

where $\tilde{M}:=\max _{x \in\left[v^{-}, v^{+}\right]} a(x)$. By virtue of (2.25) and (2.26), since $x^{\prime}(\bar{t})>0$, we get $x(+\infty)-x(\bar{t})=\int_{\bar{t}}^{+\infty} x^{\prime}(t) \mathrm{d} t=+\infty$, in contradiction with the boundedness of $x$.

Therefore, $x^{\prime}(t) \equiv 0$ in $[\tilde{t},+\infty)$ and by the definition of $\tilde{t}$ this implies $\tilde{t}=t^{*}$. So, $x^{\prime}(t) \equiv$ 0 in $\left[t^{*},+\infty\right)$ and by the definition of $t^{*}$ this implies $t^{*}=L$.

Remark 2.7. In view of what observed in Remark 6 [13], if the sign condition in (2.29) is satisfied with the reverse inequality, i.e., if

$$
\operatorname{tf}(t, x, y) \geq 0 \text { for a.e. }|t| \geq L, \quad \text { every } x \in \mathbb{R},|y|<\rho,
$$

then it is possible to prove that $\lim _{x \rightarrow \pm \infty} x^{\prime}(t)=0$ and $x^{\prime}(t) \leq 0$ for $|t| \geq L$. So, since $v^{-}<$ $v^{+}$, when $L=0$ problem $(P)$ does not admit solutions. 


\section{Criteria for right-hand side of the type $f(t, x, y)=b(t, x) c(x, y)$}

In this section we present some operative criteria useful when the right-hand side has the following product structure

$$
f(t, x, y)=b(t, x) c(x, y)
$$

As we will show, there is a strict link between the local behaviors of $c(x, \cdot)$ at $y=0$ and of $b(\cdot, x)$ at infinity which plays a key role for the existence or non-existence of solutions.

In what follows we assume that $b$ is a Carathéodory function and $c$ is a continuous function satisfying

$$
c(x, y)>0 \quad \text { for every } y \neq 0 \text { and } x \in\left[v^{-}, v^{+}\right] ; \quad c\left(v^{-}, 0\right)=c\left(v^{+}, 0\right)=0 .
$$

Notice that in this framework, the constant functions $\alpha(t): \equiv v^{-}$and $\beta(t): \equiv v^{+}$are a pair of well-ordered, monotone, lower and upper solutions. Consequently, according to the notations given after Definition 2.1, in this case we have

$$
\mathcal{I}=\left[v^{-}, v^{+}\right], \quad v=v^{+}-v^{-}, \quad d=0
$$

and again

$$
m:=\min _{x \in \mathcal{I}} a(x)>0, \quad M:=\max _{x \in \mathcal{I}} a(x),
$$

According to the results of the previous section, the first three results provide sufficient conditions for the existence of solutions for our special $f$ split in the product of $b$ and $c$. Then we will deal with sufficient conditions for the non-existence of solutions.

Theorem 3.1. Let there exists a function $\lambda \in L_{\text {loc }}^{q}(\mathbb{R}), 1 \leq q \leq+\infty$, such that

$$
|b(t, x)| \leq \lambda(t) \quad \text { for a.e. } t \in \mathbb{R}, \quad \text { every } x \in\left[v^{-}, v^{+}\right] .
$$

Suppose that there exist positive constants $h_{1}, h_{2}, k_{1}, k_{2}, \rho, H, L, \varepsilon$, with $\varepsilon \leq 1$, and a constant $\sigma \in\left[-1,-1+\frac{h_{1} k_{1}}{M} \varepsilon\right)$, such that for every $x \in\left[v^{-}, v^{+}\right]$we have

$$
\begin{aligned}
& t \cdot b(t, x) \leq 0 \quad \text { for a.e. }|t|>L, \\
& h_{1}|t|^{-1} \leq|b(t, x)| \leq h_{2}|t|^{\sigma}, \quad \text { for a.e. }|t|>L, \\
& k_{1} \Phi(|y|) \leq c(x, y) \leq k_{2} \Phi(|y|)^{\varepsilon}, \quad \text { whenever }|y|<\rho, \\
& c(x, y) \leq k_{2}|\Phi(y)|^{2-\frac{1}{q}} \quad \text { whenever }|y| \geq H .
\end{aligned}
$$

Finally, let conditions (2.2) and (2.3) hold with $0<\mu<\frac{h_{1} k_{1}}{M}$.

Then, problem $(P)$ admits solutions.

Proof. Put $\theta(r):=k_{2}\left(\frac{r}{m}\right)^{2-\frac{1}{q}}$ for $r>0$, from (3.1) and (3.5) it is immediate to verify the validity of conditions (2.4) and (2.5). Let us now fix a constant $C>0$ and put

$$
\hat{C}:=\max \left\{\rho, \Phi^{-1}\left(\frac{M}{m} \Phi(C)\right)\right\} \text {. }
$$


Since $c(x, y)>0$ for $y \neq 0$, denoted by $\hat{m}_{C}:=\min \left\{c(x, y): x \in\left[v^{-}, v^{+}\right], \rho \leq|y| \leq \hat{C}\right\}$, we have $\hat{m}_{C}>0$. Finally, put

$$
\psi_{C}:=\min \left\{\frac{\hat{m}_{C}}{\Phi(\hat{C})}, k_{1}\right\}
$$

Consider the following functions:

$$
\begin{aligned}
\gamma(t) & :=\min \left\{\min _{x \in\left[v^{-}, \nu^{+}\right]}|b(-t, x)|, \min _{x \in\left[v^{-}, v^{+}\right]}|b(t, x)|\right\}, \quad t \geq 0 ; \\
H_{C}(t) & := \begin{cases}0 & \text { for } 0 \leq t \leq L ; \\
\psi_{C} \int_{L}^{t} \gamma(\tau) \mathrm{d} \tau & \text { for } t \geq L ;\end{cases} \\
M_{C}(t) & :=\Phi^{-1}\left(\frac{M}{m} \Phi(C) e^{-\frac{1}{M} H_{C}(|t|)}\right), \quad t \in \mathbb{R} .
\end{aligned}
$$

Observe that by assumption (3.3) we have $\gamma(t)>0$ for a.e. $t \geq L$ and $\lim _{t \rightarrow+\infty} H_{C}(t)=+\infty$, hence $\lim _{|t| \rightarrow+\infty} M_{C}(t)=0$. So, there exists a constant $L_{C}^{*}>L$ such that $M_{C}(t) \leq \rho$ whenever $|t| \geq L_{C}^{*}$. Let us define

$$
K_{C}(t):= \begin{cases}H_{C}(t) & \text { for } 0 \leq t \leq L_{C^{\prime}}^{*} \\ H_{C}\left(L_{C}^{*}\right)+k_{1} \int_{L_{C}^{*}}^{t} \gamma(\tau) \mathrm{d} \tau & \text { for } t>L_{C}^{*}\end{cases}
$$

and let $N_{C}$ be the function defined in (2.7).

By the positivity of the function $\gamma, K_{C}$ is strictly increasing for $t \geq L$. Moreover, by condition (3.1), we have $K_{C} \in W_{l o c}^{1,1}([0,+\infty))$. Further, since $\psi_{C} \leq k_{1}$ we have $H_{C}(t) \leq$ $K_{C}(t)$ for every $t \geq 0$ and then $N_{C}(t) \leq M_{C}(t)$ for every $t \in \mathbb{R}$.

Observe that by (3.2) and the definition of $\psi_{C}$, we obtain

$$
f(t, x, y)=b(t, x) c(x, y) \leq \psi_{C} b(t, x) \Phi(|y|) \leq-K_{C}^{\prime}(t) \Phi(|y|)
$$

and

$$
f(-t, x, y)=b(-t, x) c(x, y) \geq \psi_{C} b(-t, x) \Phi(|y|) \geq K_{C}^{\prime}(t) \Phi(|y|)
$$

for a.e. $t \in\left(L, L_{C}^{*}\right)$, every $x \in\left[v^{-}, v^{+}\right]$and every $|y| \leq N_{C}(t) \leq \hat{C}$. Similarly, by (3.4) we have

$$
f(t, x, y)=b(t, x) c(x, y) \leq k_{1} b(t, x) \Phi(|y|) \leq-K_{C}^{\prime}(t) \Phi(|y|)
$$

and

$$
f(-t, x, y)=b(-t, x) c(x, y) \geq k_{1} b(-t, x) \Phi(|y|) \geq K_{C}^{\prime}(t) \Phi(|y|)
$$

for a.e. $t \geq L_{C}^{*}$, every $x \in\left[v^{-}, v^{+}\right]$and every $|y| \leq N_{C}(t) \leq M_{C}(t) \leq \rho$. Then, condition (2.8) of Theorem 2.3 holds.

Now, from (3.3) it follows that $h_{1} k_{1} t^{-1} \leq K_{C}^{\prime}(t)$ for a.e. $t \geq L_{C}^{*}$. As a consequence,

$$
K_{C}(t) \geq K_{C}\left(L_{C}^{*}\right)+h_{1} k_{1} \log \frac{t}{L_{C}^{*}} \quad \text { for every } t>L_{C}^{*} .
$$

Then, by the upper bound on the exponent $\mu$ we get

$$
\int^{+\infty} e^{-\frac{1}{\mu M} K_{C}(t)} \mathrm{d} t \leq \text { Const. } \int^{+\infty} t^{-\frac{h_{1} k_{1}}{\mu M}} \mathrm{~d} t<+\infty,
$$


and condition (2.6) follows.

Finally, let us define

$$
\eta_{C}(t):= \begin{cases}\max _{x \in\left[\nu^{-}, v^{+}\right]}|b(t, x)| \cdot \max _{(x, y) \in\left[v^{-}, \nu^{+}\right] \times[-\hat{C}, \hat{C}]} c(x, y) & \text { if }|t| \leq L_{C}^{*} \\ h_{2} k_{2}|t|^{\sigma} \Phi\left(N_{C}(t)\right)^{\varepsilon} & \text { if }|t|>L_{C}^{*} .\end{cases}
$$

By (3.3) and (3.4), for every $y \in \mathbb{R}$ such that $|y| \leq N_{C}(t)$ for a.e. $t \in \mathbb{R}$ and every $x \in$ $\left[v^{-}, v^{+}\right]$, it results

$$
|f(t, x, y)|=|b(t, x)| c(x, y) \leq \eta_{C}(t),
$$

that is condition (2.9), so it remains to prove that $\eta_{C} \in L^{1}(\mathbb{R})$. To this purpose, notice that by (3.1) and the continuity of $c$ we have $\eta_{C} \in L^{1}\left(\left[-L_{C^{\prime}}^{*} L_{C}^{*}\right]\right)$. Moreover, when $|t|>L_{C}^{*}$, by (3.6) we have

$$
0<\eta_{C}(t) \leq h_{2} k_{2}|t|^{\sigma}\left(\frac{M}{m} \Phi(C)\right)^{\varepsilon} e^{-\frac{\varepsilon}{M} K_{C}(|t|)} \leq \text { Const. }|t|^{\sigma-\frac{h_{1} k_{1}}{M} \varepsilon} .
$$

Since $\sigma<\frac{h_{1} k_{1}}{M} \varepsilon-1$, we get $\eta_{C} \in L^{1}(\mathbb{R})$. Therefore, Theorem 2.3 applies and guarantees the assertion of the present result.

For differential operators having superlinear growth at infinity, the following result can be applied, whose proof is a consequence of Theorem 2.4.

Theorem 3.2. Let all the assumptions of Theorem 3.1 be satisfied with the exception of (2.2) and with (3.5) replaced by

$$
\lim _{|y| \rightarrow+\infty} \frac{\max _{x \in\left[\nu^{-}, v^{+}\right]} c(x, y)}{|\Phi(y)|}=0 .
$$

Then, if (2.3) holds true with a positive $\mu<\frac{h_{1} k_{1}}{M}$, problem $(P)$ admits solutions.

Proof. Set

$$
\theta(s):=\max _{x \in\left[v^{-}, v^{+}\right]}\left(\max \left\{c\left(x, \Phi^{-1}\left(\frac{s}{a(x)}\right)\right), c\left(x, \Phi^{-1}\left(-\frac{s}{a(x)}\right)\right)\right\}\right) .
$$

Observe that $\theta$ is a continuous function on $[0,+\infty)$, such that

$$
\theta(a(x)|\Phi(y)|) \geq c(x, y) \quad \text { for every } x \in\left[v^{-}, \nu^{+}\right], y \in \mathbb{R},
$$

hence (2.4) holds. Moreover, by (3.7), for every $\varepsilon>0$ there exists a real $c_{\varepsilon}$ such that

$$
c(x, y) \leq \varepsilon|\Phi(y)| \quad \text { for every } x \in\left[v^{-}, \nu^{+}\right],|y| \geq c_{\varepsilon} .
$$

Hence, for every $s \geq M \max \left\{\Phi\left(c_{\varepsilon}\right),-\Phi\left(-c_{\varepsilon}\right)\right\}$ we have $\theta(s) \leq \frac{\varepsilon}{m} s$, that is

$$
\lim _{s \rightarrow+\infty} \frac{\theta(s)}{s}=0 .
$$

Hence, the proof proceeds as that of Theorem 3.1, applying Theorem 2.4 instead of Theorem 2.3. $\square$

Finally, in the case of $p$-Laplacian operators, the following result holds, as a consequence of Theorem 2.5, by the same proof of Theorem 3.1.

Theorem 3.3. Consider $\Phi(y)=|y|^{p-2} \mathrm{y}, p>1$, and let all the assumptions of Theorem 3.1 be satisfied with the exception of (2.2) and condition (3.5) replaced by 


$$
c(x, y) \leq k_{2}|y|^{p-\frac{1}{q}}, \quad \text { for every }|y| \geq H .
$$

Then, if $p<1+\frac{h_{1} k_{1}}{M}$, problem $(P)$ admits solutions.

Proof. Define $\theta(r):=k_{2}\left(\frac{r}{m}\right)^{1+\frac{1}{p-1}\left(1-\frac{1}{q}\right)}$. Easy computations allow to verify that assumptions (2.19) and (2.20) are satisfied. The conclusion follows as in the proof of Theorem 3.1, now applying Theorem 2.5. In fact, observe that in this case (2.3) is satisfied for $\mu=p-1$ and, defined $K_{C}$ and $\eta_{C}$ as in the proof of Theorem 3.1, conditions (2.22) and (2.23) hold true. Notice that they are the rewriting of conditions (2.8) and (2.9), respectively, in the case of $p$-Laplacian operators.

In the previous results the requirement $\mu<\frac{h_{1} k_{1}}{M}$ is not merely technical, but it is essential, as it will be clarified by the following non-existence result.

Theorem 3.4. Suppose that (3.2) holds for a.e. $t \in \mathbb{R}$ and let there exist a real constant $\Lambda>0$ and a positive function $\lambda \in L^{1}(0, \Lambda)$ such that

$$
|b(t, x)| \leq \ell(|t|) \quad \text { for a.e. }|t| \leq \Lambda, x \in\left[v^{-}, v^{+}\right] .
$$

Moreover, assume that there exist positive constants $h, k, \rho$ such that

$$
\begin{aligned}
& |b(t, x)| \leq h|t|^{-1}, \quad \text { for every } x \in \mathbb{R}, \text { a.e. }|t|>\Lambda \\
& c(x, y) \leq k \Phi(|y|), \quad \text { for every } x \in \mathbb{R}, 0<y<\rho .
\end{aligned}
$$

If (2.25) holds with a positive $\mu \geq \frac{h k}{m}$, then problem $(P)$ does not have solutions.

Proof. Put $K(t):=k \int_{0}^{t} \ell(\tau) \mathrm{d} \tau$ for $t \in[0, \Lambda]$ and $K(t):=\int_{0}^{\Lambda} \ell(\tau) \mathrm{d} \tau+h k(\log t-\log L)$ for $t \geq \Lambda$. Note that assumptions (2.27) and (2.28) are satisfied for $L=0$. Moreover,

$$
\int^{+\infty} e^{-\frac{1}{\mu m} K(t)} \mathrm{d} t \geq \text { Const. } \int^{+\infty} t^{-\frac{1}{\mu m} h k} \mathrm{~d} t=+\infty
$$

by the lower bound on the exponent $\mu$. So, the assertion follows from Theorem 2.6. 口

The following results are immediate consequences of Theorems 3.1, 3.2, and 3.4.

Corollary 3.5. Let $f(t, x, y)=h(t) g(x) c(y)$, with $h \in L_{\text {loc }}^{q}(\mathbb{R})$, for $1 \leq q \leq+\infty$, c continuous in $\mathbb{R}$ and $g$ continuous and positive in $\left[v^{-}, v^{+}\right]$.

Assume that $t \cdot h(t) \leq 0$ for every $t \in \mathbb{R}$ and $c(y)>0$ for every $y \neq 0$. Moreover, suppose that

$$
\lim _{|t| \rightarrow+\infty}|t h(t)|=: h_{1} \in(0,+\infty), \quad \lim _{|y| \rightarrow 0} \frac{c(y)}{\Phi(|y|)}=: k_{1} \in(0,+\infty) .
$$

Let (2.2) holds and

$$
\limsup _{|y| \rightarrow+\infty} \frac{c(y)}{|\Phi(y)|^{2-\frac{1}{q}}}<+\infty .
$$

Then, if (2.3) holds with an exponent $\mu$ such that $h_{1} k_{1} \cdot \min _{x \in\left[v^{-}, v^{+}\right]} g(x)>M \mu$, problem $(P)$ admits solutions; instead if $(2.25)$ holds with an exponent $\mu$ satisfying $h_{1} k_{1} \cdot \max _{x \in\left[v^{-}, v^{+}\right]} g(x)<m \mu,(P)$ does not admit solutions. 
Corollary 3.6. Let all the assumptions of Corollary 3.5 be satisfied, apart (2.2) and with (3.12) replaced by the following condition

$$
\lim _{|y| \rightarrow+\infty} \frac{c(\gamma)}{|\Phi(\gamma)|}=0 .
$$

Then, the same conclusions of Corollary 3.5 hold.

Finally, for the $p$-Laplacian operator we can state the following criterium, consequence of Theorems 3.3 and 3.4.

Corollary 3.7. Let $f(t, x, y)=h(t) g(x) c(y)$, with $h \in L_{\text {loc }}^{q}(\mathbb{R})$, for $1 \leq q \leq+\infty, b$ continuous in $\mathbb{R}, g$ continuous and positive in $\left[v^{-}, v^{+}\right]$. Let $\Phi(y)=|y|^{p-2} y$, for $p>1$.

Assume that $t \cdot h(t) \leq 0$ for every $t \in \mathbb{R}$ and $c(y)>0$ for every $y \neq 0$. Moreover, suppose that there exist

$$
\lim _{|t| \rightarrow+\infty}|t h(t)|=: h_{1}, \quad \lim _{|y| \rightarrow 0} \frac{c(y)}{|y|^{p-1}}=: k_{1}, \quad \limsup _{|y| \rightarrow+\infty} \frac{c(y)}{|y|^{p-\frac{1}{q}}}<+\infty,
$$

for a positive constant $h_{1}$.

Then, if $h_{1} k_{1} \cdot \min _{x \in\left[v^{-}, v^{+}\right]} g(x)>M(p-1)$, problem $(P)$ admits solutions; instead if $h_{1} k_{1} \cdot \max _{x \in\left[v^{-}, v^{+}\right]} g(x)<m(p-1),(P)$ does not have solutions.

We conclude with some examples in which the previous corollaries apply.

Example 3.8. Let $f(t, x, y):=-h \frac{t}{\sqrt{1+t^{4}}} g(x)|y|^{\mu}\left(1+|y|^{2}\right)^{\frac{\mu-2}{2}}$ where $h$ is a positive constant and $g$ is a generic continuous function, positive in $\left[v^{-}, v^{+}\right]$. Suppose that $\Phi(y)=$ $y|y|^{\mu-2}|\arctan y|$ with $\mu \geq 1$ for every $y \in \mathbb{R}$ and $a(x) \equiv 1$ for every $x \in \mathbb{R}$.

If $h(t):=-h \frac{t}{\sqrt{1+t^{4}}}$ and $c(y):=|y|^{\mu}\left(1+|y|^{2}\right)^{\frac{\mu-2}{2}}$, it is immediate to check that all the assumptions of Corollary 3.5 are satisfied for $q:=+\infty, h_{1}:=h, k_{1}:=1$. Then, if $\min _{x \in\left[\nu^{-}, \nu^{+}\right]} g(x)>\frac{\mu}{h}$ problem $(P)$ has solutions, instead if $\max _{x \in\left[v^{-}, v^{+}\right]} g(x)<\frac{\mu}{h}$ then problem $(P)$ does not have solutions.

Example 3.9. Let $f(t, x, y):=-h \frac{t}{\sqrt{1+t^{4}}} g(x)|y|^{\beta}$, where $h$ is a positive constant and $g$ is a generic continuous function, positive in $\left[v^{-}, v^{+}\right]$. Let $\Phi(y):=y|y|^{\beta-1} e^{|y|}$ and $a(x) \equiv 1$. Then condition (3.13) is satisfied for every $\beta>0$ and all the assumptions of Corollary 3.6 hold with $h_{1}:=h$ and $k_{1}:=1$. Then, if $\min _{x \in\left[v^{-}, \nu^{+}\right]} g(x)>\frac{\mu}{h}$ problem $(P)$ has solutions, instead if $\max _{x \in\left[v^{-}, v^{+}\right]} g(x)<\frac{\mu}{h}$ then problem $(P)$ does not have solutions.

Example 3.10. Let $f(t, x, y):=-h \frac{\arctan t}{t} g(x)|y|^{p-1} \sqrt{1+y^{2}}$, where $h$ is a positive constant and $g$ is a generic continuous function, positive in $\left[v^{-}, v^{+}\right]$. Let $\Phi(y):=y|y|^{p-2}$ and $a(x) \equiv 1$. Then all the assumptions of Corollary 3.7 are satisfied, for $q:=+\infty, h_{1}:=\frac{h \pi}{2}$, $h_{2}:=1$. Then, if $\min _{x \in\left[v^{-}, v^{+}\right]} g(x)>\frac{2(p-1)}{\pi h}$ problem $(P)$ has solutions, instead if $\max _{x \in\left[\nu^{-}, \nu^{+}\right]} g(x)<\frac{2(p-1)}{\pi h}$ then problem $(P)$ does not have solutions.

Remark 3.11. Note that in [11] the existence of heteroclinic solutions was proved when in assumption (2.8) one has $\Phi(|y|)^{\gamma}$, instead of $\Phi(|y|)$, for some $\gamma>1$. Of course, 
for small $|y|$ we have $\Phi(|y|)^{\gamma}<\Phi(|y|)$ for each $\gamma>1$, hence the present condition (2.8) implies the validity of the analogous condition with $\gamma>1$, assumed in [11] (see condition (8)). But, on the other hand, taking $\gamma>1$ one can lose the summability of the function $K_{C}$ required in assumption (7) of [11]. In fact, in the following example the present Theorems 2.3 and 2.4 are applicable, whereas the results established in [11] do not work.

Consider the problem, already discussed in Example 4 [7]:

$$
\left\{\begin{array}{l}
\left(\Phi\left(x^{\prime}(t)\right)\right)^{\prime}=m(t) \Phi\left(\left|x^{\prime}(t)\right|\right), \quad \text { a.e. on } \mathbb{R} \\
x(-\infty)=0, x(+\infty)=1,
\end{array}\right.
$$

where $a(x) \equiv 1$ and $m: \mathbb{R} \rightarrow \mathbb{R}$ is the function defined by

$$
m(t)=\left\{\begin{array}{cc}
-\frac{\alpha}{t}, & |t|>1 \\
-\alpha t, & |t| \leq 1,
\end{array}\right.
$$

for some $\alpha>0$. As it easy to check, the best function $K_{C}$ satisfying condition (8) in [11] is $K_{C}(t):=[\alpha \log t]_{+}$, but condition (7) of [11] does not hold, whatever $\gamma>1$ may be. Hence, the existence results proved in [11] are not applicable. Instead, notice that condition (2.6) herein considered holds whenever $\alpha>\mu$ (see (2.3)) and Theorem 2.3 (or Theorem 2.4) applies, provided that the operator $\Phi$ also satisfies the other required assumptions. Similar considerations can be done for the $p$-Laplacian operator too, using Theorem 2.5 .

\section{Author details}

${ }^{1}$ Dipartimento di Matematica - Università di Bologna, Piazza di Porta S.Donato 5, 40126 Bologna, Italy ${ }^{2}$ Dipartimento di Scienze Matematiche - Università Politecnica delle Marche, Via Brecce Bianche, 60131 Ancona, Italy

\section{Authors' contributions}

The authors wrote this article in collaboration and with same responsibility. All authors read and approved the final manuscript.

\section{Competing interests}

The authors declare that they have no competing interests.

Received: 22 January 2011 Accepted: 23 September 2011 Published: 23 September 2011

\section{References}

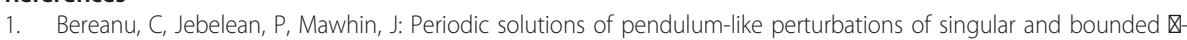
Laplacians. J Dyn Diff Equ. 22, 463-471 (2010). doi:10.1007/s10884-010-9172-3

2. Bereanu, C, Mawhin, J: Nonlinear Neumann boundary-value problems with $\Phi$-Laplacian operators. An Stiint Univ Ovidius Constanta. 12, 73-92 (2004)

3. Bereanu, C, Mawhin, J: Boundary-value problems with non-surjective $\Phi$-Laplacian and one-side bounded nonlinearity. Adv Differ Equ. 11, 35-60 (2006)

4. Bereanu, C, Mawhin, J: Existence and multiplicity results for some nonlinear problems with singular $\Phi$-Laplacian. J Differ Equ. 243, 536-557 (2007). doi:10.1016/j.jde.2007.05.014

5. Bereanu, C, Mawhin, J: Periodic solutions of nonlinear perturbations of $\Phi$-Laplacians with possibly bounded $\Phi$. Nonlinear Anal. 68, 1668-1681 (2008)

6. Bereanu, C, Mawhin, J: Boundary value problems for some nonlinear systems with singular $\Phi$-Laplacian. J Fixed Point Theory Appl. 4, 57-75 (2008). doi:10.1007/s11784-008-0072-7

7. Bianconi, B, Papalini, F: Non-autonomous boundary value problems on the real line. Discrete Contin Dyn Syst. 15, 759-776 (2006)

8. Kyritsi, ST, Matzakos, N, Papageorgiou, NS: Nonlinear boundary value problems for second order differential equations. Czech Math J. 55, 545-579 (2005). doi:10.1007/s10587-005-0046-5

9. Papageorgiou, EH, Papageorgiou, NS: Strongly nonlinear, multivalued, periodic problems with maximal monotone terms. Differ Integral Equ. 17, 443-480 (2004)

10. Papalini, F: Solvability of strongly nonlinear boundary value problems for second order differential inclusions. Nonlinear Anal. 66, 2166-2189 (2007). doi:10.1016/j.na.2006.03.007

11. Cupini, G, Marcelli, C, Papalini, F: Heteroclinic solutions of boundary value problems on the real line involving general nonlinear differential operators. Differ Integral Equ. 24, 619-644 (2011) 
12. Ferracuti, L, Papalini, F: Boundary value problems for strongly nonlinear multivalued equations involving different $\Phi$ Laplacians. Adv Differ Equ. 14, 541-566 (2009)

13. Marcelli, C, Papalini, F: Heteroclinic connections for fully nonlinear non-autonomous second order differential equations. J Differ Equ. 241, 160-183 (2007). doi:10.1016/.j.je.2007.05.038

doi:10.1186/1687-2770-2011-26

Cite this article as: Cupini et al:: On the solvability of a boundary value problem on the real line. Boundary Value Problems 2011 2011:26.

Submit your manuscript to a SpringerOpen ${ }^{\odot}$ journal and benefit from:

- Convenient online submission

- Rigorous peer review

- Immediate publication on acceptance

- Open access: articles freely available online

- High visibility within the field

- Retaining the copyright to your article

Submit your next manuscript at $\boldsymbol{\nabla}$ springeropen.com 